\section{Rebuilding from the rubble}

Three months ago, a massive earthquake hit the disputed region of Kashmir in northern Pakistan. Now that the initial images have disappeared from the media, what hope is left in their wake?

Deborah Cohen BMJ

If any benefit is to come from the devastation left after the earthquake in Kashmir, it's an opportunity to replace the area's underdeveloped healthcare system. That's according to Professor Murad Khan, a professor of psychiatry at the Aga Khan University in Karachi, who implemented a post-disaster mental health relief plan to affected areas. Although Pakistan has well developed health policies,

tated large parts of Pakistan controlled Kashmir leaving 3.3 million people homeless and plunged into poverty. Because many remote villages are without banks, people kept hard cash and jewellery in their homes; these valuables were buried as their houses collapsed. Livestock and food supplies that had been collected for the winter months were also lost. Some people were afraid to leave the area in

respiratory and gastrointestinal illnesses and other illnesses caused by the cold, the wet, and poor nutrition. People also have infected wounds owing to the poor post-operative care that they received in the overwhelmed hospitals and makeshift medical centres in the days after the initial shock. Limbs were amputated and fractures set in less than ideal conditions. Professor Khan says that estimates of the death toll will rise from 86000 to 200000 .

The poor accessibility of the region is hampering the relief operation-the already fragile infrastructure was destroyed, and the aftershocks have continued to turn roads into piles of rocks and gravel. Helicopters are the mainstay for delivering supplies and collecting sick people. But because of the terrain and

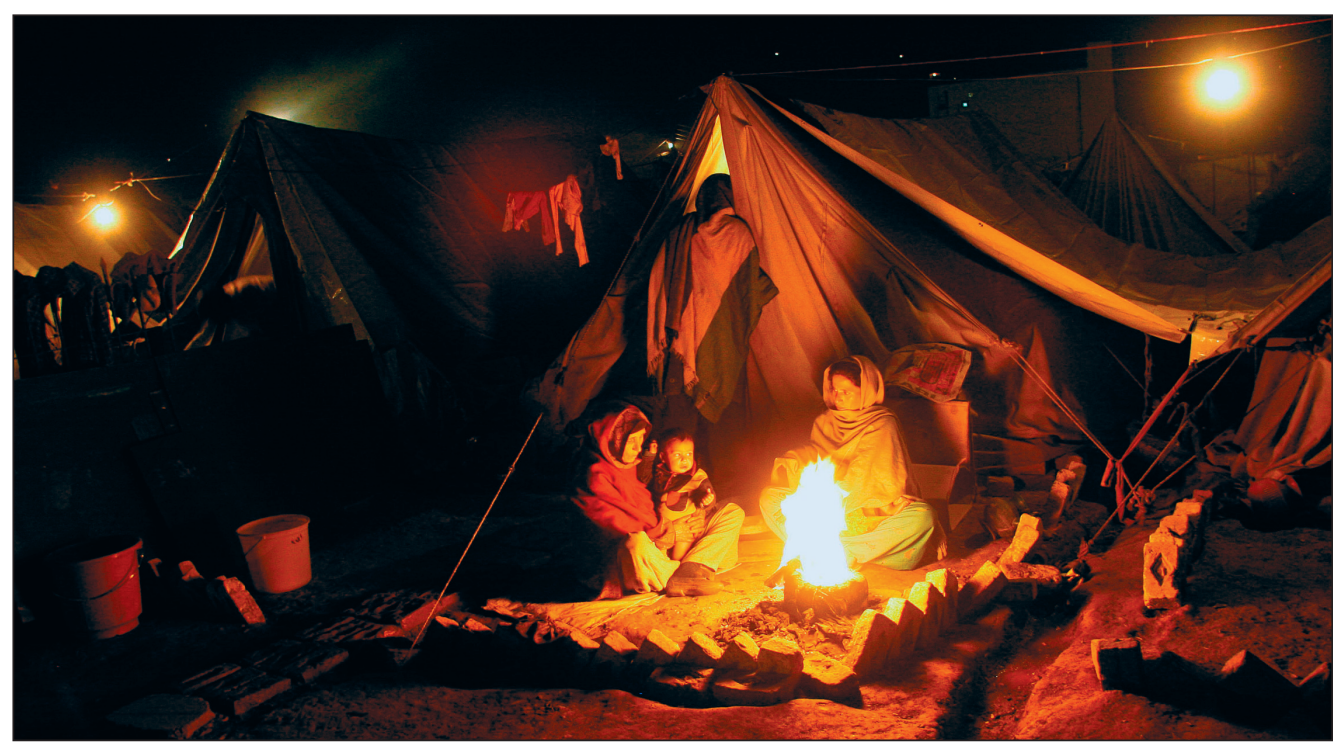

Women cook on an open fire in front of their tent in a refugee camp in Muzaffarabad, Pakistan

their effects barely reached the area hit by the earthquake on 8 October 2005.

Speaking at an international mental health meeting last month at the Institute of Psychiatry, London, he said: "It's a chance to start from the beginning. The money is available and the people are here. We've seen the goodwill from Pakistanis and the international community, and that needs to be harnessed."

Professor Khan added: "This is a big wakeup call. We must put the money and resources where they matter. We've been caught on the back foot, and, if we're not careful, the disaster will get worse."

The earthquake, measuring 7.6 on the Richter scale, devas- which their houses once stood. People have no paper deeds, and the remnants of their homes are the only evidence that support their claim to land. And other people were concerned about looting.

Because of this lack of food and shelter, relief workers are worried that a secondary disaster will occur in the coming winter, says Alan Bell, the Asia based operations manager for Merlin, the UK charity specialising in medical relief and health care: "People are left without adequate food, shelter, and medical care. These people are going to face severe hardship throughout the winter."

Mr Bell says that medical staff are seeing more and more adverse weather conditions, helicopters sometimes cannot reach the villages. Medical workers have to carry their own equipment and walk-sometimes for several days-to the stranded villages.

Kemal Chaudri, a neurosurgeon who helped to give medical relief in isolated areas, says that there is a need for basic medical care. "We walked up to the isolated areas with a guy from special forces to help us. We saw lots of elderly people and women, who because of culture wouldn't come down to the medical centres or couldn't come down because of injury. They had conditions like goitre, high blood pressure, and diabetes."

He also highlighted the need for female doctors. "There were two female doctors with us and they were inundated with [gynaecological] problems, as patients didn't want to talk to a male doctor. One of our doctors saw 400 patients a day. This was principally because she was female."

As attention has slowly turned from the immediate to the long term needs of the affected area, the impact of the earthquake has become more apparent. Not only do the towns and villages need to be reconstructed, but also people need physical, social, and psychological support. Professor Khan said that people in the area wanted someone to listen, but they did not know who to share their experiences with-everyone has suffered. Programmes tend to concentrate on conditions such as post-traumatic stress disorder and other stress reactions, but anxiety and depression are also prevalent.

"People are dealing with multiple traumas-lost family members, livelihoods, and savings. Some require long term rehabilitation, such as those with spinal injuries. Those who have had limbs amputated will require a different kind of rehabilitationin the mountainous areas, wheelchairs and crutches will not be practical. We have to think innovatively how to help these people," says Professor Khan.

The fabric of society has also been disrupted; whole generations have been wiped out. The earthquake struck during school time, and many of the children who survived have been orphaned. The Pakistani government has placed a ban on the adoption of children for six months to stop people taking children and exploiting them. "Usually extended families would absorb orphans into them. But the families have also disappeared. We need to think of ways to support them," Professor Khan says.

Professor Khan remains sceptical, however, that the government will take over after the non-governmental organisations leave. "The medical community in the Western world must collaborate to help develop health systems and capacity on the ground. Not everywhere needs a tertiary care approach. We can train health workers at the community level to deal with minor problems. There needs to be a really effective primary care system, of which mental health is an integral part." 